\title{
NOTE ON THE SHAPIRO POLYNOMIALS
}

JOHN BRILLHART AND L. CARLITZ

1. Introduction. The polynomials $P_{n}(x)$ and $Q_{n}(x)$, which we are concerned with here, were introduced in 1951 by H. S. Shapiro [5, p. 39 ] in his study of the magnitude of certain trigonometric sums. They are defined recursively by the formulas

$$
P_{n+1}(x)=P_{n}(x)+x^{2^{n}} Q_{n}(x), \quad Q_{n+1}(x)=P_{n}(x)-x^{2^{n}} Q_{n}(x),
$$

where $n \geqq 0$ and $P_{0}(x)=Q_{0}(x)=1$. (See [4] also. Note in this reference that $P_{0}(x)=Q_{0}(x)=x$.)

These polynomials have been used by Kahane and Salem in their book [1] to prove several theorems about trigonometric series. Rider [2] used a generalization of these polynomials to complete the solution of a problem partially solved in [4]. In a more recent paper Rider [3] employed the polynomials to exhibit certain subalgebras of the group algebra of the unit circle. In particular, in this paper Rider obtained a special case of Theorem 4 below.

The first few polynomials are

$$
\begin{aligned}
& P_{1}(x)=1+x, \quad P_{2}(x)=1+x+x^{2}-x^{3}, \\
& P_{3}(x)=1+x+x^{2}-x^{3}+x^{4}+x^{5}-x^{6}+x^{7}, \\
& Q_{1}(x)=1-x, \quad Q_{2}(x)=1+x-x^{2}+x^{3}, \\
& Q_{3}(x)=1+x+x^{2}-x^{3}-x^{4}-x^{5}+x^{6}-x^{7} .
\end{aligned}
$$

It is clear from this definition that $\operatorname{deg} P_{n}=\operatorname{deg} Q_{n}=2^{n}-1$.

In this note we will derive a relation between $P_{n}(x)$ and $Q_{n}(x)$ and use it to show that these polynomials have equal discriminants. We will also find a formula for the resultant of the two polynomials, and develop an explicit formula for their coefficients. The latter will then be used to compute the value of $P_{n}(x)$ at $x= \pm 1, \pm i$, and certain other points on the unit circle.

2. We begin by deriving the relation that exists between $P_{n}(x)$ and $Q_{n}(x)$.

Theorem 1. $Q_{n}(x)=(-1)^{n} x^{2^{n}-1} P_{n}(-1 / x), n \geqq 0$.

Proof. By induction. The theorem holds for $n=0,1$. Assume the relation for $n, n \geqq 1$. Then 1969.

Received by the editors February 13, 1969 and, in revised form, September 18. 


$$
\begin{aligned}
& (-1)^{n+1} x^{2^{n+1}-1} P_{n+1}(-1 / x) \\
& =(-1)^{n+1} x^{2^{n+1}-1}\left[P_{n}(-1 / x)+(-1 / x)^{2^{n}} Q_{n}(-1 / x)\right] \\
& =(-1)^{n+1} x^{2^{n+1}-1}\left[(-1)^{n} Q_{n}(x) / x^{2^{n}-1}+x^{-2^{n}}(-1)^{n}(-1 / x)^{2^{n}-1} P_{n}(x)\right] \\
& =P_{n}(x)-x^{2^{n}} Q_{n}(x)=Q_{n+1}(x) \text {. }
\end{aligned}
$$

The following properties of the discriminant $D$ of a polynomial will be of use in establishing the corollary below. Let $c \neq 0$ be a constant and $f(x)$ by a polynomial of degree $n$. Then

(i) $D(f(c x))=c^{n(n-1)} D(f(x))$.

(ii) $D(c f(x))=c^{2 n-2} D(f(x))$.

(iii) $D\left(x^{n} f(1 / x)\right)=D(f(x))$.

Corollary. $D\left(P_{n}(x)\right)=D\left(Q_{n}(x)\right), n \geqq 0$.

Proof.

$$
\begin{aligned}
D\left(Q_{n}(x)\right) & =D\left((-1)^{n} x^{2^{n}-1} P_{n}(-1 / x)\right) \\
& =D\left((-1)^{n+1} x^{2^{n}-1} P_{n}(1 / x)\right),
\end{aligned}
$$

using (i) with $c=-1$. The corollary then follows from (ii) and (iii). The first few completely factored values of $D\left(P_{n}(x)\right)$ are listed in the table below

\begin{tabular}{l|l}
$n$ & \multicolumn{1}{|c}{$D\left(P_{n}(x)\right)$} \\
\hline 1 & 1 \\
2 & $-2^{2} \cdot 11$ \\
3 & $2^{10} \cdot 5^{2} \cdot 193$ \\
4 & $2^{34} \cdot 32834009652827$
\end{tabular}

We next recall several properties of the resultant $R$ of two polynomials $f$ and $g$ of degree $n$ and $m$ respectively.

(i) $R(f, c g)=c^{n} R(f, g), c$ a constant.

(ii) $R(f, g)=a^{d} R(f, g+\lambda f)$, where $a$ is the leading coefficient of $f$, $\lambda$ is an arbitrary polynomial, and $d=\operatorname{deg} g-\operatorname{deg}(g+\lambda f)$.

(iii) $R(f, g)=(-1)^{m n} R(g, f)$.

(iv) $R(f, g h)=R(f, g) R(f, h)$.

Theorem 2. $R\left(P_{n}(x), Q_{n}(x)\right)=(-1)^{n-1} 2^{2^{n+1}-n-2}, n \geqq 1$.

Proof. For $n=1$ we have $R\left(P_{1}, Q_{1}\right)=2$. Suppose $n>1$. Then 


$$
\begin{aligned}
R\left(P_{n}, Q_{n}\right) & =R\left(P_{n-1}+x^{2^{n-1}} Q_{n-1}, P_{n-1}-x^{2^{n-1}} Q_{n-1}\right) \\
& =R\left(P_{n-1}+x^{2^{n-1}} Q_{n-1}, 2 P_{n-1}\right) \\
& =-2^{2^{n}-1} R\left(P_{n-1}, P_{n-1}+x^{2^{n-1}} Q_{n-1}\right) \\
& =-2^{2^{n}-1} R\left(P_{n-1}, x^{2^{n-1}} Q_{n-1}\right) \\
& =-2^{2^{n}-1} R\left(P_{n-1}, x^{2^{n-1}}\right) R\left(P_{n-1}, Q_{n-1}\right) .
\end{aligned}
$$

But $R\left(P_{n-1}, x^{2^{n-1}}\right)=1$. Hence $R\left(P_{n}, Q_{n}\right)=-2^{2^{n}-1} R\left(P_{n-1}, Q_{n-1}\right)$. From this reduction step, used repeatedly, we obtain the evaluation $R\left(P_{n}, Q_{n}\right)=\left\{\prod_{s=2}^{n}\left(-2^{2^{s}-1}\right)\right\} R\left(P_{1}, Q_{1}\right)=(-1)^{n-1} 2^{2^{n+1}-n-2}$.

The next theorem permits the generation of $P_{n}(x)$ and $Q_{n}(x)$ without combining the two types of polynomials.

THEOREM 3.

$$
\begin{aligned}
& P_{n+1}(x)=P_{n}\left(x^{2}\right)+x P_{n}\left(-x^{2}\right), \quad n \geqq 0 . \\
& Q_{n+1}(x)=Q_{n}\left(x^{2}\right)+x Q_{n}\left(-x^{2}\right), \quad n \geqq 1 \text {. }
\end{aligned}
$$

Proof. By induction. The formulas are true for $n=0,1$. Assume both formulas hold for $n, n \geqq 1$. Then

$$
\begin{aligned}
P_{n+1}(x) & =P_{n}(x)+x^{2^{n}} Q_{n}(x) \\
& =\left[P_{n-1}\left(x^{2}\right)+x P_{n-1}\left(-x^{2}\right)\right]+x^{2^{n}}\left[Q_{n-1}\left(x^{2}\right)+x Q_{n-1}\left(-x^{2}\right)\right] \\
& =\left[P_{n-1}\left(x^{2}\right)+x^{2^{n}} Q_{n-1}\left(x^{2}\right)\right]+x\left[P_{n-1}\left(-x^{2}\right)+x^{2^{n}} Q_{n-1}\left(-x^{2}\right)\right] .
\end{aligned}
$$

Hence,

$$
P_{n+1}(x)=P_{n}\left(x^{2}\right)+x P_{n}\left(-x^{2}\right) .
$$

The formula for $Q_{n+1}(x)$ is established in a similar manner.

3. We now turn to an investigation of the coefficients of $P_{n}(x)$. (The corresponding results can be obtained for $Q_{n}(x)$ through the use of Theorem 1.)

It is clear from (1) that $P_{n}(x)$ has coefficients \pm 1 , without gaps, and that the first $2^{n}$ coefficients of $P_{n+1}(x)$ are identical with those of $P_{n}(x)$. It follows then that these coefficients do not depend on $n$, so we can write $P_{n}(x)=\sum_{r=0}^{2^{n}-1} a(r) x^{r}, n \geqq 0$. (We may, of course, also consider $P_{n}(x)$ as the first $2^{n}$ terms of the infinite series $P_{\infty}(x)$ $=\sum_{r=0}^{\infty} a(r) x^{r}$.)

We will now derive an explicit formula for $a(r)$.

THEOREM 4. If we write $r=r_{0}+r_{1} \cdot 2+r_{2} \cdot 2^{2}+\cdots+r_{k} \cdot 2^{k}, k \geqq 0$, $r_{i}=0$ or 1 , then 


$$
a(r)=(-1)^{r_{0} r_{1}+r_{1} r_{2}+\cdots+r_{k-1} r_{k}} .
$$

Proof. We observe in (2) that the even and odd degree terms on the right side are separated, which allows us to equate coefficients, obtaining the relations $a(2 r)=a(r)$ and $a(2 r+1)=(-1)^{r} a(r)$. If we write $a(r)=(-1)^{e(r)}$, then

$$
e(2 r) \equiv e(r) \quad \text { and } \quad e(2 r+1) \equiv r+e(r)(\bmod 2) .
$$

Proceeding by induction on $k$, we verify for $k=0$ that $1=a\left(r_{0}\right)$ $=(-1)^{0}$, where $r_{0}=0$ or 1 . Assume next that $e(r)=r_{1} r_{2}+r_{2} r_{3}+\cdots+$ $r_{k-1} r_{k}$ for any $r=r_{1}+r_{2} \cdot 2+\cdots+r_{k} \cdot 2^{k-1}$ of $k$ digits. Consider the number $2 r+r_{0}$, where $r_{0}=0$ or 1 . Then using (4) $e\left(2 r+r_{0}\right) \equiv r_{0} r+e(r)$ $\equiv r_{0} r_{1}+e(r)=r_{0} r_{1}+r_{1} r_{2}+\cdots+r_{k-1} r_{k}(\bmod 2)$. (Note the particular case $a\left(2^{t}\right)=1$.)

4. We next consider the problem of evaluating $P_{n}(x)$ at certain points on the unit circle. We begin with

THEOREM 5.

$$
\begin{aligned}
& P_{2 n}(1)=2^{n}, \quad P_{2 n+1}(1)=2^{n+1}, \quad n \geqq 0 . \\
& P_{2 n}(-1)=2^{n}, \quad P_{2 n+1}(-1)=0, \quad n \geqq 0 .
\end{aligned}
$$

Proof. Let $\theta(n)$ be the number of $a(r)$ in $P_{n}(x)$ that are positive. In particular, let $\theta_{0}(n)$ be the number of $a(2 r)$ and $\theta_{1}(n)$ be the number of $a(2 r+1)$ in $P_{n}(x)$ that are positive. Then certainly

$$
\theta(n)=\theta_{0}(n)+\theta_{1}(n) .
$$

Since the first term on the right side of (2) contains all the terms of even degree, we have

$$
\theta_{0}(n+1)=\theta(n)
$$

and hence by (5)

$$
\theta_{0}(n+1)=\theta_{0}(n)+\theta_{1}(n) .
$$

Also, since the second term on the right side of (2) can be written as $\sum_{r=0}^{2^{n}-1}(-1)^{r} a(r) x^{2 r+1}$, we find that

$$
\theta_{1}(n+1)=\theta_{0}(n)+\left[2^{n-1}-\theta_{1}(n)\right] .
$$

Adding this equation to (7), and using (5), we obtain

$$
\theta(n+1)=\theta_{0}(n+1)+\theta_{1}(n+1)=2 \theta_{0}(n)+2^{n-1} .
$$

Finally, from (6) we derive the recursion relation 


$$
\theta(n+1)=2 \theta(n-1)+2^{n-1} .
$$

With the initial conditions $\theta(0)=1$, and $\theta(1)=2$, the solution is readily found to be

$$
\theta(2 n)=2^{2 n-1}+2^{n-1}, \quad \theta(2 n+1)=2^{2 n}+2^{n}, \quad n \geqq 0 .
$$

From the equation $P_{n}(1)=\theta(n)-\left[2^{n}-\theta(n)\right]=2 \theta(n)-2^{n}$, we conclude that $P_{2 n}(1)=2^{n}$ and $P_{2 n+1}(1)=2^{n+1}$. If we now set $x=1$ in (2), we have $P_{n}(-1)=P_{n+1}(1)-P_{n}(1)$, whence $P_{2 n}(-1)=2^{n}$ and $P_{2 n+1}(-1)=0$.

With a knowledge of $P_{n}( \pm 1)$, we are in a position to find the values at $x=e^{\pi i / 2^{t}}$. For example, setting $x=i$ in (2), we obtain $P_{n+1}(i)=P_{n}(-1)+i P_{n}(1)$, whence $P_{2 n}(i)=i \cdot 2^{n}$ and $P_{2 n+1}(i)=(1+i) 2^{n}$. The values at $x=-i$ are found by conjugating.

REMARK. It can readily be shown by repeated use of (2) that the series $P_{\infty}(x)$ diverges at the dense set of points $\exp \left(2 \pi r i / 2^{s}\right)$ on the unit circle.

The authors would like to thank Michael Garvey for his suggestions on parts of the paper.

\section{REFERENCES}

1. J. Kahane and R. Salem, Ensembles parfaits et séries trigonométriques, Actualités Sci. Indust., no. 1301, Hermann, Paris, 1963, p. 134. MR 28 \#3279.

2. D. Rider, Transformations of Fourier coefficients, Pacific J. Math 19 (1966), 347-355. MR 34 \#3195.

3. - Closed subalgebras of $L^{1}(T)$, Duke Math. J. 36 (1969), 105-115.

4. W. Rudin, Some theorems on Fourier coefficients, Proc. Amer. Math. Soc. 10 (1959), 855-859. MR 22 \#6979.

5. H. S. Shapiro, Extremal problems for polynomials and power series, M.I.T. Master's Thesis, Cambridge, Mass., 1951.

University of Arizona, Tucson, Arizona 85721 and

Duke University, Durham, North Carolina 27706 